J. Environ. Sci. \& Natural Resources, 5(1): 319-328, 2012

ISSN 1999-7361

\title{
Natural Food Abundance and SIS Biodiversity of Matshaya Rani Fish Sanctuary
}

\author{
M. A. N. G. M. Hasan, N. S. Lucky and M. A. R. Hossain
}

Department of Fisheries Biology and Genetics, Bangladesh Agricultural University, Mymensingh

\begin{abstract}
An investigation was carried out in the Matshaya Rani (fish queen) Fish sanctuary to evaluate the hydrographic status, natural food and SIS (small indigenous species) abundance in the old Brahmaputra River near the BAU campus. There were three treatment sites- inside sanctuary, upstream and downstream of the sanctuary named as treatments $\mathrm{T}_{1}, \mathrm{~T}_{2}$ and $\mathrm{T}_{3}$, respectively. $\mathrm{A}$ number of water quality parameters such as temperature $\left({ }^{0} \mathrm{C}\right)$, transparency $(\mathrm{cm})$, depth $(\mathrm{cm})$, water velocity $\left(\mathrm{m} \mathrm{sec}{ }^{-1}\right)$, $\mathrm{pH}$, dissolved oxygen $\left(\mathrm{mg} \mathrm{l}^{-1}\right)$, conductivity $\left(\mu \mathrm{cm}^{-1}\right)$, TDS $\left(\mathrm{mg} \mathrm{l}^{-1}\right)$, alkalinity $\left(\mathrm{mg} \mathrm{l}^{-1}\right)$, phosphate-phosphorus (mg $\left.\mathrm{l}^{-1}\right)$, nitratenitrogen $\left(\mathrm{mg} \mathrm{l}^{-1}\right)$, ammonia-nitrogen $\left(\mathrm{mg} \mathrm{l}^{-1}\right)$ and chlorophyll- $a\left(\mu \mathrm{g}^{-1}\right)$ were recorded and were found within the suitable range for natural food and SIS in different treatments. Among all the water quality parameters, only water transparency and depth showed significant difference $(\mathrm{P}<0.05)$ among the treatments. Forty three genera of phytoplankton belonging to Bacillariophyceae $(10)$, Chlorophyceae (22), Cyanophyceae (8), and Euglenophyceae (3) and nine genera of zooplankton were identified belonging to Rotifera (4) and Crustacea (5) in all the treatments. None of plankton group except Cyanophyceae and Rotifera showed significant difference $(\mathrm{p}<0.05)$ among the treatments. Thirty two species of SIS under ten groups were found in different treatments. The highest number of species was found in treatment $T_{1}(30)$ followed by $T_{2}(25)$ and $T_{3}(24)$. The catfishes, loaches and minnows dominated among thirty two species caught from the sampling sites. The highest numbers of species were found in April and May and the least species were found the month of January. Significant inter month and inter site variation $(\mathrm{P}<0.05)$ in numerical abundance of fish observed in the study area. Notable endangered fishes, Kanchan puti- (Puntius conchonius), Balichata (Acanthocobitis botia), Indian torrent catfish-Amblyceps mangois and Chaka- (Chaca chaca) species were only found in Matshaya Rani Fish sanctuary. The natural food abundance and SIS diversity were significantly more in the inside of Matshaya Rani Fish sanctuary than both the upstream and downstream of the sanctuary.
\end{abstract}

Key words: Conservation, Endangered fish, Fish biodiversity, Pollution

\section{Introduction}

The small indigenous species (SIS) of fish in Bangladesh are generally considered to be those which grow to a length of about $25 \mathrm{~cm}$ or 9 inches at maturity (Hossain et al., 1991 and 1999). They are also known as "miscellaneous species of fish and prawn". Small indigenous fish species (SIS) have high nutritional value in terms of protein, micronutrients, vitamins and minerals, and these micronutrients and minerals are not commonly available in other foods. Prior to 1960, many different small indigenous fish species were abundant in the rivers, borropits, roadsides, canals, streams, beels, jheels and ponds of Bangladesh. Since 1960's, the biodiversity of small indigenous fish species has been in decline despite their ability to reproduce quickly in poor environmental condition. As $80 \%$ of the rural people mostly depend on SIS, the research will directly benefit the targeted marginal farmers, retailers and indirectly to numerous.

Production from open water is gradually declining because of over-fishing, siltation, pollution and myopic management practices and so on. A good number of natural fishes which are highly regarded for their taste and nutritive value are now endangered. To protect the native species different development projects attempted to establish fish sanctuaries in the open water bodies in different areas of the country. Although establishing sanctuary in open waters is a well-known method for conservation of biodiversity in the open waters, the information on size, seasonality, katha materials, fish preference, local knowledge on sanctuaries are very scarce under Bangladesh context. Study is essential to know the number and weight of fish species, yearly production, relative abundance of fish and impacts of fish sanctuary on production and biodiversity of fish in rivers and beels. The distribution of the fish and other fisheries fauna are accelerated by different types of limnological as well as hydrographic status viz. temperature, transparency, depth, velocity, sunshine, $\mathrm{pH}$, dissolved oxygen, etc. The production of natural food organisms especially different types of plankton depend on hydrographic status. Since planktons furnish direct food of fishes, precise information on the species composition of planktons and their seasonal variations are of special importance to proper fisheries management. It is, therefore, imperative to undertake a detailed study of the composition of the planktonic flora and fauna in relation to various physical, chemical and biological conditions. Very little work has been done in Bangladesh on the dynamic aspects of plankton and other qualitative values of water, establishment of 
fish sanctuaries, and conservation of SIS and keeps the biodiversity sustainable from generation to generation. During 2005-2006, the Matshaya Rani Fish Sanctuary were developed through people's participation for enhancing production, maintaining biodiversity of fish and other aquatic flora and fauna and improving the livelihood of the poor fishers.

The distribution of fish depends on the natural food availability, environmental variables and other factors (Whitefield et al., 1981). Despite many studies, little information is available on fish specially SIS abundance inside and outside sanctuary in open water bodies of Bangladesh. However, the proposed study was carried out to evaluate the hydrographic status, natural food and SIS abundance in the Matshaya Rani Fish sanctuary and compared with the same parameters upstream and downstream of the sanctuary.

\section{Materials and Methods}

\section{Site selection}

The study was conducted for a period of five months, from January to May in Matshaya Rani Fish sanctuary which was established on 1 January 2008 in a section of the river old Brahmaputra beside the BAU campus under Mymensingh district. The sanctuary area is about $200 \mathrm{~m} \times 25 \mathrm{~m}$, and the water depth increases up to $9.14 \mathrm{~m}$ to $15.24 \mathrm{~m}$ during the monsoon months and goes down to $3.04 \mathrm{~m}$ in the dry months.

\section{Experimental design}

For the present study, three treatments were used namely, Matshaya Rani fish sanctuary for treatment $\left(\mathrm{T}_{1}\right)$, upstream of the river $(1 \mathrm{~km}$ distance from the sanctuary) as treatment $\left(\mathrm{T}_{2}\right)$ and downstream of the river (1 km distance from the sanctuary) as treatment $\left(\mathrm{T}_{3}\right)$ having three replicates of each treatments.

\section{Analysis of water quality parameters}

A number of water quality parameters such as temperature $(\mathrm{C})$, transparency $(\mathrm{cm})$, depth $(\mathrm{cm})$, water velocity $(\mathrm{m} / \mathrm{s}), \mathrm{pH}$, dissolved oxygen $(\mathrm{mg} / \mathrm{l})$, conductivity $(\mu \mathrm{s} / \mathrm{cm})$, TDS (mg/l), alkalinity (mg/l), phosphate-phosphorus (mg/l), nitrate-nitrogen $(\mathrm{mg} / \mathrm{l})$, ammonia-nitrogen $(\mathrm{mg} / \mathrm{l})$ and chlorophyll-a $(\mu \mathrm{g} / \mathrm{l})$ were recorded monthly. Temperature $\left({ }^{0} \mathrm{C}\right)$, transparency $(\mathrm{cm}), \mathrm{pH}$ and dissolved oxygen $(\mathrm{mg} / \mathrm{l})$ were measured on the spot and rests of the above parameters were measured at the Water Quality and Pond Dynamics Laboratory in the Department of Fisheries Management, Bangladesh Agricultural University, Mymensingh.

\section{Sampling of plankton}

Plankton samples were collected monthly from each treatment. Twenty liters of water sample was taken from different places and depths of the river and passed through fine $(25 \mu)$ mesh plankton net. Filtered samples was taken into a measuring cylinder and carefully made up to a standard volume of $50 \mathrm{ml}$. Then the collected plankton samples were preserved in $5 \%$ buffered formalin in small plastic bottles each for subsequent studies.

From each $50 \mathrm{ml}$ preserved sample, $1 \mathrm{ml}$ sub-sample was examined by using a Sedge Wick-Rafter cell (S$\mathrm{R}$ cell) and a binocular microscope (Olympus $\mathrm{CH}-40$ ) with phase contrast facilities. One $\mathrm{ml}$ of sub sample was transferred to the S-R cell and then all planktonic organisms present in 10 squares of the cell chosen randomly were identified and counted. Plankton identification was performed following APHA (1992). The quantitative estimation of plankton was done using the following formula:

$$
\mathrm{N}=\frac{\mathrm{A} \times 1000 \times \mathrm{C}}{\mathrm{V} \times \mathrm{F} \times \mathrm{L}}
$$

Where, $\mathrm{N}$ is the number of plankton cells or units per liter of original water, $\mathrm{A}$ is the total number of plankton counted, $\mathrm{C}$ is the volume of final concentrate sample in $\mathrm{ml}, \mathrm{V}$ is the volume of field in cubic $\mathrm{mm}, \mathrm{F}$ is the number of field counted and $\mathrm{L}$ is the volume of original water in liter. For each treatment, mean number of plankton was recorded and expressed numerically in per liter of water.

\section{Sampling of fish}

Fish samples were collected monthly from the three treatments of the Matshaya Rani. For the collection of fish samples a bamboo made fishing trap, locally called ' $U$ cha' with 9 feet length and 7 feet wide and a mesh size of 2- $4 \mathrm{~mm}$ was used. Three Ucha were set inside the sanctuary and three Ucha were set in the river at the upstream and downstream of the sanctuary each. Every month, the individual length and weight of the collected fishes was measured by using a measurement scale and a digital electronic balance (KERN, Model No. EMB 2000-0), respectively. The morphometric characteristics of each sample were also observed to identify the fish species. Total numbers of each collected species were counted and then released carefully into the sanctuary. Fish samples were also collected from 1 $\mathrm{km}$ distance in both sides of the sanctuary (up and down) to understand the monthly fluctuation in fish catch. 


\section{J. Environ. Sci. \& Natural Resources, 5(1): 319-328, 2012}

\section{Results}

\section{Water quality parameters}

Water quality parameters were analyzed to observe any appreciable changes that might have occurred in response to different treatments. The results of the water quality analysis indicated the suitable ranges of quality parameters for natural food and SIS in treatment $\mathrm{T}_{1}, \mathrm{~T}_{2}$ and $\mathrm{T}_{3}$ (Table 1).

Table 1. Water quality parameters as obtained under three treatments during the study period, in mean \pm standard error.

\begin{tabular}{|c|c|c|c|c|}
\hline \multirow[t]{2}{*}{ Parameter } & \multicolumn{3}{|c|}{ Treatments } & \multirow{2}{*}{$\begin{array}{c}\text { Level of } \\
\text { significance }\end{array}$} \\
\hline & $\mathrm{T}_{1}$ & $\mathrm{~T}_{2}$ & $\mathrm{~T}_{3}$ & \\
\hline $\begin{array}{l}\text { Surface } \\
\text { Temperature }\left({ }^{\circ} \mathrm{C}\right)\end{array}$ & $\begin{array}{c}25.50 \pm 1.54 \\
(15-32)\end{array}$ & $\begin{array}{c}25.04 \pm 1.38 \\
(15-31)\end{array}$ & $\begin{array}{c}24.49 \pm 1.35 \\
(15-30)\end{array}$ & NS \\
\hline $\begin{array}{l}\text { Bottom Temperature } \\
\left({ }^{\circ} \mathrm{C}\right)\end{array}$ & $\begin{array}{l}24.32 \pm 1.26 \\
(16-30.00)\end{array}$ & $\begin{array}{l}24.61 \pm 1.32 \\
(16-30.20)\end{array}$ & $\begin{array}{c}24.19 \pm 1.35 \\
(15-30)\end{array}$ & NS \\
\hline Transparency $(\mathrm{cm})$ & $\begin{array}{c}175.20^{\mathrm{a}} \pm 5.89 \\
(141-208)\end{array}$ & $\begin{array}{c}84.4000^{\mathrm{b}} \pm 2.27993 \\
(77-99)\end{array}$ & $\begin{array}{c}82.6000^{\mathrm{b}} \pm 2.06051 \\
(70-95)\end{array}$ & * \\
\hline Velocity $(\mathrm{m} / \mathrm{s})$ & $\begin{array}{l}0.12 \pm 0.003 \\
(0.10-0.14)\end{array}$ & $\begin{array}{l}0.12 \pm 0.001 \\
(0.10-0.12)\end{array}$ & $\begin{array}{l}0.11 \pm 0.001 \\
(0.10-0.12)\end{array}$ & NS \\
\hline Depth $(\mathrm{cm})$ & $\begin{array}{c}256.20^{\mathrm{a}} \pm 3.86 \\
(220-276)\end{array}$ & $\begin{array}{c}93.53^{\mathrm{b}} \pm 2.80 \\
(79-110)\end{array}$ & $\begin{array}{c}91.80^{\mathrm{b}} \pm 2.69 \\
(75-106)\end{array}$ & * \\
\hline $\begin{array}{l}\text { Conductivity } \\
(\mu \mathrm{s} / \mathrm{cm})\end{array}$ & $\begin{array}{l}251.80 \pm 6.12 \\
\quad(202.77-286)\end{array}$ & $\begin{array}{c}248.00 \pm 6.46 \\
(202-278)\end{array}$ & $\begin{array}{c}243.20 \pm 6.67 \\
(202-278)\end{array}$ & NS \\
\hline TDS (mg/l) & $\begin{array}{c}159.89 \pm 1.84 \\
(145-170)\end{array}$ & $\begin{array}{c}160.50 \pm 1.39 \\
(149-171)\end{array}$ & $\begin{array}{c}159.92 \pm 1.55 \\
(149-171)\end{array}$ & NS \\
\hline $\mathrm{pH}$ & $\begin{array}{l}8.113 \pm 0.093 \\
(7.30-8.70)\end{array}$ & $\begin{array}{l}8.126 \pm 0.091 \\
(7.40-8.60)\end{array}$ & $\begin{array}{l}7.973 \pm 0.076 \\
(7.40-8.40)\end{array}$ & NS \\
\hline Surface DO $(\mathrm{mg} / \mathrm{l})$ & $\begin{array}{l}6.740 \pm 0.351 \\
(5.28-8.45)\end{array}$ & $\begin{array}{l}6.794 \pm 0.357 \\
(5.30-8.39)\end{array}$ & $\begin{array}{c}6.676 \pm 0.328 \\
(5.30-8.39)\end{array}$ & NS \\
\hline Bottom DO (mg/l) & $\begin{array}{c}6.663 \pm 0.321 \\
(5.34-8.40)\end{array}$ & $\begin{array}{l}6.678 \pm 0.322 \\
(5.29-8.43)\end{array}$ & $\begin{array}{l}6.678 \pm .032 \\
(5.29-8.43)\end{array}$ & $\mathrm{NS}$ \\
\hline $\mathrm{NO}_{3}-\mathrm{N}(\mathrm{mg} / \mathrm{l})$ & $\begin{array}{c}0.0407 \pm 0.00345 \\
(0.02-0.06)\end{array}$ & $\begin{array}{c}0.0507 \pm 0.00371 \\
(0.03-0.07)\end{array}$ & $\begin{array}{c}0.0407 \pm 0.00248 \\
(0.03-0.06)\end{array}$ & NS \\
\hline $\mathrm{PO}_{4}-\mathrm{P}(\mathrm{mg} / \mathrm{l})$ & $\begin{array}{c}0.226 \pm 0.018 \\
(0.12-0.35)\end{array}$ & $\begin{array}{c}0.275 \pm 0.042 \\
(0.17-0.83)\end{array}$ & $\begin{array}{c}0.229 \pm 0.009 \\
(0.15-0.29)\end{array}$ & NS \\
\hline $\mathrm{NH}_{3}-\mathrm{N}(\mathrm{mg} / \mathrm{l})$ & $\begin{array}{c}0.200 \pm 0.018 \\
(0.11-0.37)\end{array}$ & $\begin{array}{l}0.222 \pm 0.026 \\
(0.10-0.44)\end{array}$ & $\begin{array}{c}0.206 \pm 0.017 \\
(0.12-0.35)\end{array}$ & NS \\
\hline Alkalinity (mg/l) & $\begin{array}{c}133.800 \pm 2.338 \\
(118-148)\end{array}$ & $\begin{array}{c}132.466 \pm 2.074 \\
(117-144)\end{array}$ & $\begin{array}{c}135.933 \pm 1.837 \\
(125-146)\end{array}$ & NS \\
\hline Chlorophyll-a $(\mu \mathrm{g} / \mathrm{l})$ & $\begin{array}{c}63.372 \pm 5.943 \\
(30-113.5)\end{array}$ & $\begin{array}{l}87.398 \pm 32.916 \\
(28.80-234.88)\end{array}$ & $\begin{array}{c}63.573 \pm 6.984 \\
(30.50-120.30)\end{array}$ & NS \\
\hline
\end{tabular}

$* \mathrm{P}<0.05$ and $\mathrm{NS}=$ Not significant

\section{Plankton populations}

Forty three genera of phytoplankton belonging to four major groups: Bacillariophyceae, Chlorophyceae, Cyanophyceae, and Euglenophyceae were identified during the study period. Nine genera of zooplankton belonging to Rotifera and Crustacea group were also identified. The mean abundance of plankton populations with their different groups are shown in Table 2. 
Table 2. Mean abundance \pm SE of plankton $\left(\times 10^{3}\right.$ cells $\left.1^{-1}\right)$ as recorded from the fish sanctuary under different treatments

\begin{tabular}{|c|c|c|c|c|}
\hline \multirow[t]{2}{*}{ Plankton group } & \multicolumn{3}{|c|}{ Treatments } & \multirow{2}{*}{$\begin{array}{c}\text { Level of } \\
\text { significance }\end{array}$} \\
\hline & $\overline{\mathrm{T}_{1}}$ & $\mathrm{~T}_{2}$ & $\overline{T_{3}}$ & \\
\hline Bacillariophyceae & $\begin{array}{c}24.433 \pm 8.34 \\
(11-139.5)\end{array}$ & $\begin{array}{c}31.500 \pm 9.32 \\
(6.5-119.5)\end{array}$ & $\begin{array}{c}30.033 \pm 9.22 \\
(5.5-119)\end{array}$ & NS \\
\hline Chlorophyceae & $\begin{array}{c}42.966 \pm 3.76 \\
(15.5-63)\end{array}$ & $\begin{array}{c}38.666 \pm 4.41 \\
(13.5-73)\end{array}$ & $\begin{array}{c}37.800 \pm 4.27 \\
(12.5-70)\end{array}$ & NS \\
\hline Cyanophyceae & $\begin{array}{c}11.466^{\mathrm{a}} \pm 1.23 \\
(4-21)\end{array}$ & $\begin{array}{c}8.4666^{b} \pm .93 \\
(4-18.5)\end{array}$ & $\begin{array}{c}7.666^{b} \pm 0.91 \\
(3.5-17.5)\end{array}$ & * \\
\hline Euglenophyceae & $\begin{array}{c}9.600 \pm 1.61 \\
(3-28.5)\end{array}$ & $\begin{array}{c}8.100 \pm 1.56 \\
(2-19.5)\end{array}$ & $\begin{array}{c}7.466 \pm 1.44 \\
(2-18)\end{array}$ & NS \\
\hline Total Phytoplankton & $\begin{array}{c}88.467 \pm 10.12 \\
(48.5-219)\end{array}$ & $\begin{array}{c}86.733 \pm 14.00 \\
(34-217)\end{array}$ & $\begin{array}{c}82.967 \pm 13.61 \\
(31.5-212)\end{array}$ & NS \\
\hline Rotifera & $\begin{array}{c}1.0000^{b} \pm 0.23 \\
(0-3.5)\end{array}$ & $\begin{array}{c}1.7333^{\mathrm{b}} \pm 0.33 \\
(0.5-4)\end{array}$ & $\begin{array}{c}8.666^{\mathrm{a}} \pm 0.15 \\
(0-2)\end{array}$ & $*$ \\
\hline Crustacea & $\begin{array}{c}2.366 \pm 0.40 \\
(0-5.5)\end{array}$ & $\begin{array}{c}2.500 \pm 0.18 \\
(1.5-4)\end{array}$ & $\begin{array}{c}2.133 \pm 0.16 \\
(1-3)\end{array}$ & NS \\
\hline Total Zooplankton & $\begin{array}{c}3.366^{b} \pm 0.49 \\
(0-5.5)\end{array}$ & $\begin{array}{c}4.233^{\mathrm{b}} \pm 0.46 \\
(2-7.5)\end{array}$ & $\begin{array}{c}10.779^{\mathrm{a}} \pm 0.24 \\
(1-4.5)\end{array}$ & $*$ \\
\hline Total Plankton & $\begin{array}{c}91.833 \pm 10.28 \\
(49.5-224)\end{array}$ & $\begin{array}{c}90.961 \pm 14.32 \\
(37-223.5)\end{array}$ & $\begin{array}{c}85.966 \pm 13.56 \\
(35-214)\end{array}$ & NS \\
\hline
\end{tabular}

Means with the different superscripts are significantly different $(P<0.05)$

\section{SIS biodiversity}

A total of 32 species were recorded from different treatments viz. 30 from Matshyarani fish sanctuary and 25 and 24 species from $1 \mathrm{~km}$ upstream and downstream of the sanctuary, respectively. The national biodiversity status of fish species that were found in the study sites is shown in Table 3. The number of SIS fluctuated in the sampling months in all the sites. Analysis of Variance (ANOVA) indicates inter-month, inter site and their interaction is significant $(P<0.05)$ in SIS numerical abundance (Table 4). Most abundant SIS in weight (top five in each treatment) in different treatments is presented in (Table 5).

Table 3. The SIS and prawn species found in different treatments of the studied sites over 5 months of the sampling period in 2008 and their national biodiversity status (Red Book, IUCN, 2000). CR: Critically endangered; EN: Endangered; VU: Vulnerable; NO: Not threatened and DD: Data deficient

\begin{tabular}{|c|c|c|c|c|c|c|c|c|}
\hline $\begin{array}{l}\text { Local } \\
\text { name }\end{array}$ & Scientific name & $\begin{array}{c}\text { IUCN BD } \\
\text { status }\end{array}$ & Treatments & Jan & Feb & Mar & Apr & May \\
\hline \multirow[t]{3}{*}{ Biam } & \multirow{3}{*}{$\begin{array}{l}\text { Mastacembelus } \\
\text { armatus }\end{array}$} & \multirow[t]{3}{*}{ EN } & $\overline{\mathrm{T}_{1}}$ & $\sqrt{ }$ & $\sqrt{ }$ & $\sqrt{ }$ & $\sqrt{ }$ & $\sqrt{ }$ \\
\hline & & & $\mathrm{T}_{2}$ & $\sqrt{ }$ & $\sqrt{ }$ & $\sqrt{ }$ & $\sqrt{ }$ & $\sqrt{ }$ \\
\hline & & & $\mathrm{T}_{3}$ & $\sqrt{ }$ & $\sqrt{ }$ & $\sqrt{ }$ & $\sqrt{ }$ & $\sqrt{ }$ \\
\hline \multirow[t]{3}{*}{ Baila } & \multirow{3}{*}{$\begin{array}{l}\text { Glossogobius } \\
\text { giuris }\end{array}$} & \multirow[t]{3}{*}{$\mathrm{NO}$} & $\overline{T_{1}}$ & $\sqrt{ }$ & $\sqrt{ }$ & $\sqrt{ }$ & $\sqrt{ }$ & $\sqrt{ }$ \\
\hline & & & $\mathrm{T}_{2}$ & $\sqrt{ }$ & $\sqrt{ }$ & $\sqrt{ }$ & $\sqrt{ }$ & $\sqrt{ }$ \\
\hline & & & $\mathrm{T}_{3}$ & $\sqrt{ }$ & $\sqrt{ }$ & $\sqrt{ }$ & $\sqrt{ }$ & $\sqrt{ }$ \\
\hline \multirow[t]{3}{*}{ Gulsha } & \multirow[t]{3}{*}{ Mystus bleekeri } & \multirow[t]{3}{*}{$\mathrm{NO}$} & $\mathrm{T}_{1}$ & $\sqrt{ }$ & $\sqrt{ }$ & $\sqrt{ }$ & $\sqrt{ }$ & $\sqrt{ }$ \\
\hline & & & $\mathrm{T}_{2}$ & $\sqrt{ }$ & $\sqrt{ }$ & $\sqrt{ }$ & $\sqrt{ }$ & $\sqrt{ }$ \\
\hline & & & $\mathrm{T}_{3}$ & $\sqrt{ }$ & $\sqrt{ }$ & $\sqrt{ }$ & $\sqrt{ }$ & $\sqrt{ }$ \\
\hline
\end{tabular}


Table 3. (cont'd)

\begin{tabular}{|c|c|c|c|c|c|c|c|c|}
\hline Local name & Scientific name & $\begin{array}{c}\text { IUCN } \\
\text { BD status }\end{array}$ & Treatments & Jan & Feb & Mar & Apr & May \\
\hline \multirow[t]{3}{*}{ Jat puti } & \multirow[t]{3}{*}{ Puntius sophore } & \multirow[t]{3}{*}{$\mathrm{NO}$} & $\overline{\mathrm{T}_{1}}$ & $\sqrt{ }$ & $\sqrt{ }$ & $\sqrt{ }$ & $\sqrt{ }$ & $\sqrt{ }$ \\
\hline & & & $\mathrm{T}_{2}$ & $\sqrt{ }$ & $\sqrt{ }$ & $\sqrt{ }$ & $\sqrt{ }$ & $\sqrt{ }$ \\
\hline & & & $\overline{T_{3}}$ & $\sqrt{ }$ & $\sqrt{ }$ & $\sqrt{ }$ & $\sqrt{ }$ & $\sqrt{ }$ \\
\hline \multirow[t]{3}{*}{ Tit puti } & \multirow[t]{3}{*}{ Puntitus ticto } & \multirow[t]{3}{*}{$\mathrm{VU}$} & $\overline{\mathrm{T}_{1}}$ & $\sqrt{ }$ & $\sqrt{ }$ & $\sqrt{ }$ & $\sqrt{ }$ & $\sqrt{ }$ \\
\hline & & & $\mathrm{T}_{2}$ & $\sqrt{ }$ & $\sqrt{ }$ & $\sqrt{ }$ & $\sqrt{ }$ & $\sqrt{ }$ \\
\hline & & & $\mathrm{T}_{3}$ & $\sqrt{ }$ & $\sqrt{ }$ & $\sqrt{ }$ & $\sqrt{ }$ & $\sqrt{ }$ \\
\hline \multirow[t]{3}{*}{ Chirka } & \multirow{3}{*}{$\begin{array}{l}\text { Macrognathus } \\
\text { pancalus }\end{array}$} & \multirow[t]{3}{*}{$\overline{\mathrm{EN}}$} & $\mathrm{T}_{1}$ & $\sqrt{ }$ & $\sqrt{ }$ & $\sqrt{ }$ & $\sqrt{ }$ & $\sqrt{ }$ \\
\hline & & & $\mathrm{T}_{2}$ & $\sqrt{ }$ & $\sqrt{ }$ & $\sqrt{ }$ & $\sqrt{ }$ & $\sqrt{ }$ \\
\hline & & & $\overline{T_{3}}$ & $\sqrt{ }$ & $\sqrt{ }$ & $\sqrt{ }$ & $\sqrt{ }$ & $\sqrt{ }$ \\
\hline \multirow[t]{3}{*}{ Chela } & \multirow{3}{*}{$\begin{array}{l}\text { Salmostoma } \\
\text { phulo }\end{array}$} & \multirow[t]{3}{*}{$\mathrm{NO}$} & $\mathrm{T}_{1}$ & $\sqrt{ }$ & $\sqrt{ }$ & $\sqrt{ }$ & $\sqrt{ }$ & $\sqrt{ }$ \\
\hline & & & $\mathrm{T}_{2}$ & & $\sqrt{ }$ & $\sqrt{ }$ & $\sqrt{ }$ & $\sqrt{ }$ \\
\hline & & & $\mathrm{T}_{3}$ & & $\sqrt{ }$ & $\sqrt{ }$ & $\sqrt{ }$ & $\sqrt{ }$ \\
\hline \multirow[t]{3}{*}{ Chaka } & \multirow[t]{3}{*}{ Chaca chaca } & \multirow[t]{3}{*}{$\mathrm{EN}$} & $\mathrm{T}_{1}$ & & & & $\sqrt{ }$ & $\sqrt{ }$ \\
\hline & & & $\mathrm{T}_{2}$ & & & & & \\
\hline & & & $\mathrm{T}_{3}$ & & & & & \\
\hline \multirow[t]{3}{*}{ Mola } & \multirow{3}{*}{$\begin{array}{l}\text { Amblypharyngod } \\
\text { on mola }\end{array}$} & \multirow[t]{3}{*}{$\mathrm{NO}$} & $\mathrm{T}_{1}$ & $\sqrt{ }$ & $\sqrt{ }$ & $\sqrt{ }$ & $\sqrt{ }$ & $\sqrt{ }$ \\
\hline & & & $\mathrm{T}_{2}$ & $\sqrt{ }$ & $\sqrt{ }$ & $\sqrt{ }$ & $\sqrt{ }$ & $\sqrt{ }$ \\
\hline & & & $\mathrm{T}_{3}$ & $\sqrt{ }$ & $\sqrt{ }$ & $\sqrt{ }$ & $\sqrt{ }$ & $\sqrt{ }$ \\
\hline \multirow[t]{3}{*}{ Kutakanti } & \multirow[t]{3}{*}{ Hara hara } & \multirow[t]{3}{*}{$\mathrm{NO}$} & $\mathrm{T}_{1}$ & $\sqrt{ }$ & $\sqrt{ }$ & $\sqrt{ }$ & $\sqrt{ }$ & $\sqrt{ }$ \\
\hline & & & $\mathrm{T}_{2}$ & & & $\sqrt{ }$ & $\sqrt{ }$ & $\sqrt{ }$ \\
\hline & & & $\mathrm{T}_{3}$ & & & $\sqrt{ }$ & $\sqrt{ }$ & $\sqrt{ }$ \\
\hline \multirow[t]{3}{*}{ Napit koi } & \multirow[t]{3}{*}{ Badis badis } & \multirow[t]{3}{*}{ EN } & $\mathrm{T}_{1}$ & & $\sqrt{ }$ & $\sqrt{ }$ & $\sqrt{ }$ & $\sqrt{ }$ \\
\hline & & & $\mathrm{T}_{2}$ & & & $\sqrt{ }$ & $\sqrt{ }$ & $\sqrt{ }$ \\
\hline & & & $\mathrm{T}_{3}$ & & & $\sqrt{ }$ & $\sqrt{ }$ & $\sqrt{ }$ \\
\hline \multirow[t]{3}{*}{ Kholisha } & \multirow{3}{*}{$\begin{array}{l}\text { Polyacanthus } \\
\text { fasciatus }\end{array}$} & $\mathrm{NO}$ & $\mathrm{T}_{1}$ & $\sqrt{ }$ & $\sqrt{ }$ & $\sqrt{ }$ & $\sqrt{ }$ & $\sqrt{ }$ \\
\hline & & & $\mathrm{T}_{2}$ & & $\sqrt{ }$ & $\sqrt{ }$ & $\sqrt{ }$ & $\sqrt{ }$ \\
\hline & & & $\mathrm{T}_{3}$ & & $\sqrt{ }$ & $\sqrt{ }$ & $\sqrt{ }$ & $\sqrt{ }$ \\
\hline Kakila & Xenentodon & $\mathrm{NO}$ & $\mathrm{T}_{1}$ & $\sqrt{ }$ & $\sqrt{ }$ & $\sqrt{ }$ & $\sqrt{ }$ & $\sqrt{ }$ \\
\hline & cancila & & $\mathrm{T}_{2}$ & $\sqrt{ }$ & $\sqrt{ }$ & $\sqrt{ }$ & $\sqrt{ }$ & $\sqrt{ }$ \\
\hline & & & $\mathrm{T}_{3}$ & $\sqrt{ }$ & $\sqrt{ }$ & $\sqrt{ }$ & $\sqrt{ }$ & $\sqrt{ }$ \\
\hline Tengra & & $\mathrm{NO}$ & $\mathrm{T}_{1}$ & $\sqrt{ }$ & $\sqrt{ }$ & $\sqrt{ }$ & $\sqrt{ }$ & $\sqrt{ }$ \\
\hline & Mystus vittatus & & $\mathrm{T}_{2}$ & $\sqrt{ }$ & $\sqrt{ }$ & $\sqrt{ }$ & $\sqrt{ }$ & $\sqrt{ }$ \\
\hline & & & $\mathrm{T}_{3}$ & $\sqrt{ }$ & $\sqrt{ }$ & $\sqrt{ }$ & $\sqrt{ }$ & $\sqrt{ }$ \\
\hline Dhela & Osteobrama cotio & $\mathrm{EN}$ & $\mathrm{T}_{1}$ & & $\sqrt{ }$ & $\sqrt{ }$ & $\sqrt{ }$ & $\sqrt{ }$ \\
\hline & cotio & & $\mathrm{T}_{2}$ & & & $\sqrt{ }$ & $\sqrt{ }$ & $\sqrt{ }$ \\
\hline & & & $\mathrm{T}_{3}$ & & & $\sqrt{ }$ & $\sqrt{ }$ & $\sqrt{ }$ \\
\hline
\end{tabular}


Table 3. (cont'd)

\begin{tabular}{|c|c|c|c|c|c|c|c|c|}
\hline Local name & Scientific name & $\begin{array}{c}\text { IUCN BD } \\
\text { status }\end{array}$ & Treatments & Jan & Feb & Mar & Apr & May \\
\hline \multirow[t]{3}{*}{ Kanpona } & \multirow{3}{*}{$\begin{array}{l}\text { Aplocheilus panchax } \\
\text { panchax }\end{array}$} & \multirow[t]{3}{*}{$\mathrm{NO}$} & $\overline{\mathrm{T}_{1}}$ & & $\sqrt{ }$ & $\sqrt{ }$ & $\sqrt{ }$ & $\sqrt{ }$ \\
\hline & & & $\mathrm{T}_{2}$ & & $\sqrt{ }$ & $\sqrt{ }$ & $\sqrt{ }$ & $\sqrt{ }$ \\
\hline & & & $\mathrm{T}_{3}$ & & $\sqrt{ }$ & $\sqrt{ }$ & $\sqrt{ }$ & $\sqrt{ }$ \\
\hline \multirow[t]{3}{*}{ Gutum } & \multirow{3}{*}{$\begin{array}{l}\text { Lepidocephalichthys } \\
\text { guntea }\end{array}$} & \multirow[t]{3}{*}{$\mathrm{NO}$} & $\mathrm{T}_{1}$ & & $\sqrt{ }$ & $\sqrt{ }$ & $\sqrt{ }$ & $\sqrt{ }$ \\
\hline & & & $\mathrm{T}_{2}$ & & $\sqrt{ }$ & $\sqrt{ }$ & $\sqrt{ }$ & $\sqrt{ }$ \\
\hline & & & $\mathrm{T}_{3}$ & & $\sqrt{ }$ & $\sqrt{ }$ & $\sqrt{ }$ & $\sqrt{ }$ \\
\hline \multirow{3}{*}{$\begin{array}{l}\text { Gang } \\
\text { gutum }\end{array}$} & \multirow{3}{*}{$\begin{array}{l}\text { Lepidocephalichthys } \\
\text { irrorata }\end{array}$} & \multirow[t]{3}{*}{$\mathrm{EN}$} & $\mathrm{T}_{1}$ & & & $\sqrt{ }$ & $\sqrt{ }$ & $\sqrt{ }$ \\
\hline & & & $\mathrm{T}_{2}$ & & & & $\sqrt{ }$ & $\sqrt{ }$ \\
\hline & & & $\mathrm{T}_{3}$ & & & & $\sqrt{ }$ & $\sqrt{ }$ \\
\hline \multirow[t]{3}{*}{ Bheda } & \multirow[t]{3}{*}{ Nandus nandus } & \multirow[t]{3}{*}{$\mathrm{VU}$} & $\mathrm{T}_{1}$ & & & $\sqrt{ }$ & $\sqrt{ }$ & $\sqrt{ }$ \\
\hline & & & $\mathrm{T}_{2}$ & & & & $\sqrt{ }$ & $\sqrt{ }$ \\
\hline & & & $\mathrm{T}_{3}$ & & & & $\sqrt{ }$ & $\sqrt{ }$ \\
\hline \multirow{3}{*}{$\begin{array}{l}\text { Savon } \\
\text { korika }\end{array}$} & \multirow[t]{3}{*}{ Schistura savona } & \multirow[t]{3}{*}{$\mathrm{NO}$} & $\mathrm{T}_{1}$ & & & $\sqrt{ }$ & $\sqrt{ }$ & $\sqrt{ }$ \\
\hline & & & $\mathrm{T}_{2}$ & & & & $\sqrt{ }$ & $\sqrt{ }$ \\
\hline & & & $\mathrm{T}_{3}$ & & & & $\sqrt{ }$ & $\sqrt{ }$ \\
\hline \multirow[t]{3}{*}{ Panga } & \multirow[t]{3}{*}{ Pangio pangia } & \multirow[t]{3}{*}{$\mathrm{NO}$} & $\mathrm{T}_{1}$ & & & & $\sqrt{ }$ & \\
\hline & & & $\mathrm{T}_{2}$ & & & & & \\
\hline & & & $\mathrm{T}_{3}$ & & & & & \\
\hline \multirow[t]{3}{*}{ Balichata } & \multirow[t]{3}{*}{ Acanthocobitis botia } & \multirow[t]{3}{*}{$\mathrm{NO}$} & $\overline{\mathrm{T}_{1}}$ & & & & $\sqrt{ }$ & $\sqrt{ }$ \\
\hline & & & $\mathrm{T}_{2}$ & & & & & \\
\hline & & & $\mathrm{T}_{3}$ & & & & & \\
\hline \multirow{3}{*}{$\begin{array}{l}\text { Kanchan } \\
\text { puti }\end{array}$} & \multirow[t]{3}{*}{ Puntius conchonius } & \multirow[t]{3}{*}{$\mathrm{EN}$} & $\mathrm{T}_{1}$ & & & & & $\sqrt{ }$ \\
\hline & & & $\mathrm{T}_{2}$ & & & & & \\
\hline & & & $\mathrm{T}_{3}$ & & & & & \\
\hline \multirow{3}{*}{$\begin{array}{l}\text { Chhep } \\
\text { chela }\end{array}$} & \multirow[t]{3}{*}{ Chela cachius } & $\mathrm{EN}$ & $\mathrm{T}_{1}$ & & & & $\sqrt{ }$ & $\sqrt{ }$ \\
\hline & & & $\mathrm{T}_{2}$ & & & & & \\
\hline & & & $\mathrm{T}_{3}$ & & & & & \\
\hline Chanda & Chanda nama & $\mathrm{VU}$ & $\mathrm{T}_{1}$ & & & & $\sqrt{ }$ & $\sqrt{ }$ \\
\hline & & & $\mathrm{T}_{2}$ & & & & $\sqrt{ }$ & $\sqrt{ }$ \\
\hline & & & $\mathrm{T}_{3}$ & & & & $\sqrt{ }$ & $\sqrt{ }$ \\
\hline Ranga & Parambassis ranga & $\mathrm{VU}$ & $\mathrm{T}_{1}$ & & & & $\sqrt{ }$ & $\sqrt{ }$ \\
\hline chanda & & & $\mathrm{T}_{2}$ & & & & & \\
\hline & & & $\overline{T_{3}}$ & & & & & \\
\hline Potka & Tetraodon cutcutia & $\mathrm{NO}$ & $\mathrm{T}_{1}$ & & & & $\sqrt{ }$ & $\sqrt{ }$ \\
\hline & & & $\mathrm{T}_{2}$ & & & & $\sqrt{ }$ & $\sqrt{ }$ \\
\hline & & & $\mathrm{T}_{3}$ & & & & $\sqrt{ }$ & $\sqrt{ }$ \\
\hline
\end{tabular}


Table 3. (cont'd)

\begin{tabular}{|c|c|c|c|c|c|c|c|c|}
\hline Local name & Scientific name & $\begin{array}{c}\text { IUCN BD } \\
\text { status }\end{array}$ & Treatments & Jan & Feb & Mar & Apr & May \\
\hline \multirow{3}{*}{$\begin{array}{l}\text { Indiant } \\
\text { catfish }\end{array}$} & \multirow{3}{*}{$\begin{array}{l}\text { Amblyceps } \\
\text { mangois }\end{array}$} & \multirow[t]{3}{*}{$\overline{\mathrm{DD}}$} & $\overline{\mathrm{T}_{1}}$ & & & $\sqrt{ }$ & $\sqrt{ }$ & $\sqrt{ }$ \\
\hline & & & $\mathrm{T}_{2}$ & & & & & \\
\hline & & & $\mathrm{T}_{3}$ & & & & & \\
\hline \multirow[t]{3}{*}{ Kucha chingri } & \multirow{3}{*}{$\begin{array}{l}\text { Machrobrachium } \\
\text { rude }\end{array}$} & \multirow[t]{3}{*}{$\mathrm{NO}$} & $\overline{\mathrm{T}_{1}}$ & $\sqrt{ }$ & $\sqrt{ }$ & $\sqrt{ }$ & $\sqrt{ }$ & $\sqrt{ }$ \\
\hline & & & $\mathrm{T}_{2}$ & $\sqrt{ }$ & $\sqrt{ }$ & $\sqrt{ }$ & $\sqrt{ }$ & $\sqrt{ }$ \\
\hline & & & $\mathrm{T}_{3}$ & $\sqrt{ }$ & $\sqrt{ }$ & $\sqrt{ }$ & $\sqrt{ }$ & $\sqrt{ }$ \\
\hline \multirow[t]{3}{*}{ Chhotka chingri } & \multirow{3}{*}{$\begin{array}{l}\text { Macrobrachium } \\
\text { malcolmsonii }\end{array}$} & \multirow[t]{3}{*}{$\mathrm{NO}$} & $\mathrm{T}_{1}$ & & & $\sqrt{ }$ & $\sqrt{ }$ & $\sqrt{ }$ \\
\hline & & & $\mathrm{T}_{2}$ & & & $\sqrt{ }$ & $\sqrt{ }$ & $\sqrt{ }$ \\
\hline & & & $\mathrm{T}_{3}$ & & & $\sqrt{ }$ & $\sqrt{ }$ & $\sqrt{ }$ \\
\hline \multirow[t]{3}{*}{ Air } & \multirow[t]{3}{*}{ Sperata aor } & \multirow[t]{3}{*}{$\mathrm{VU}$} & $\overline{\mathrm{T}_{1}}$ & & & & & \\
\hline & & & $\mathrm{T}_{2}$ & & & & $\sqrt{ }$ & $\sqrt{ }$ \\
\hline & & & $\mathrm{T}_{3}$ & & & & & \\
\hline \multirow[t]{3}{*}{ Rani } & \multirow[t]{3}{*}{ Botia daryo } & \multirow[t]{3}{*}{$\overline{E N}$} & $\mathrm{~T}_{1}$ & & & & & \\
\hline & & & $\mathrm{T}_{2}$ & $\sqrt{ }$ & & $\sqrt{ }$ & $\sqrt{ }$ & $\sqrt{ }$ \\
\hline & & & $\mathrm{T}_{3}$ & & $\sqrt{ }$ & $\sqrt{ }$ & $\sqrt{ }$ & $\sqrt{ }$ \\
\hline
\end{tabular}

Table 4. ANOVA of the effects of month, site and interaction on numerical abundance of SIS in the river Brahmaputra from January to May 2008 (DF, degree of freedom; SS, sum of square; MS, Mean square; SS/df; F, MS among/MS within).

\begin{tabular}{|l|c|c|c|c|}
\hline Source & SS & DF & MS & F-value \\
\hline Month & 275.186 & 4 & 68.796 & $5.096^{* * *}$ \\
\hline Sampling site & 349.678 & 2 & 174.839 & $12.951^{* * *}$ \\
\hline Month $\times$ Sampling site & 375.147 & 8 & 46.893 & $3.474^{* * * *}$ \\
\hline
\end{tabular}

Table 5. Weight (gm) of fish in different Sampling sites (mean weight \pm standard error)

\begin{tabular}{|l|c|c|c|}
\hline \multirow{2}{*}{$\begin{array}{c}\text { Most abundant } \\
\text { species }\end{array}$} & \multicolumn{3}{|c|}{ Weight of fish in Sampling site } \\
\cline { 2 - 4 } & Sanctuary $\left(\mathrm{T}_{1}\right)$ & Upstream $\left(\mathrm{T}_{2}\right)$ & Downstream $\left(\mathrm{T}_{3}\right)$ \\
\hline Baim & $263.55 \pm 42.12(1)$ & $83.52 \pm 14.78(3)$ & $86.4 \pm 13.55(3)$ \\
\hline Baila & $142.53 \pm 21.83(3)$ & $89.18 \pm 14.33(2)$ & $97.17 \pm 15.58(2)$ \\
\hline Gulsa & $257.11 \pm 52.98(2)$ & $123.1 \pm 20.97(1)$ & $118.73 \pm 17.95(1)$ \\
\hline Tengra & $96.69 \pm 14.74(5)$ & - & - \\
\hline Chotka chingri & $112.22 \pm 18.16(4)$ & $62.77 \pm 16.03(4)$ & $59.4 \pm 16.08(4)$ \\
\hline Jat punti & - & $51.94 \pm 10.68(5)$ & $59.1 \pm 7.24(5)$ \\
\hline
\end{tabular}

Values in parenthesis indicate the abundance ranking of SIS in each treatment

\section{Discussion}

The growth of aquatic organisms strongly depends on the water quality. In the present study we investigated all physical and chemical factors of water quality parameters of Matshyarani Fish sanctuary. The temperature of the Matshyarani fish sanctuary was found to vary from 15 to $32^{\circ} \mathrm{C}$, which supposed to be suitable for growth of primary and secondary 
producers and also for fish. Similar results were also found by the Nath and Srivastava (2001). In the present study the transparency ranges from 70 to 208 $\mathrm{cm}$ was recorded which is similar with the study of CIFRI (2000) who recorded water transparency ranging from 60 to $200 \mathrm{~cm}$. The highest transparency observed in treatment $T_{1}$ might be due to the highest depth. The velocity of different treatments was also varied from 0.10 to $0.14 \mathrm{~m} / \mathrm{s}$ during the study period. The maximum velocity of $0.14 \mathrm{~m} / \mathrm{s}$ was found during March in treatment $\mathrm{T}_{1}$ while the minimum velocity of $0.10 \mathrm{~m} / \mathrm{s}$ was found during April in all the treatments. Nath and Srivastava (2001) reported that the water depth of Narmada River was moderate during postmonsoon period (3.0-15.0), which was reduced in winter (1.3-5) and was very low during summer. The present study was also observed a significant difference in depth with months among the treatments. Matshyarani sanctuary was built in the deeper place and the diversity of species depends primarily upon the nature of environment. The TDS of different treatments were varied from 145 to 171 $\mathrm{mg} / \mathrm{l}$ during the study period. Hoq et al. (2006) revealed that 20-220 mg/l of TDS were observed in the Sundarbans water of Bangladesh. DoF (1996) reported that the range of $\mathrm{pH}$ of a suitable water body would be 6.5-8.5. In the present study, $\mathrm{pH}$ values varied from 7.3 to 8.7 , which was similar to the findings of Nath and Srivastava (2001) and CIFRI (2000). The concentrations of dissolved oxygen in the experimental sites were generally fluctuated and having the range from 5.28 to $8.45 \mathrm{mg} \mathrm{l}^{-1}$, which were similar to the study of CIFRI (2000). In the present study, dissolved oxygen concentrations were suitable for fish culture throughout the experimental period. The range of $\mathrm{NO}_{3}-\mathrm{N}$ from 0.022 to $0.078 \mathrm{mg} \mathrm{l}^{-1}$ was found in open water by Pathak et al. (2001) that is similar with the findings of this study. The major source of ammonia in pond water is the direct excretion of ammonia by fish (Tucker and Boyd, 1979). In the present study, the highest and the lowest concentration of total ammonia were 0.10 to $0.44 \mathrm{mg}$ $\mathrm{l}^{-1}$, respectively. Rahman (2000) recorded $\mathrm{NH}_{3}-\mathrm{N}$ of 0.15 to $0.18 \mathrm{mg} \mathrm{L}^{-1}$ in Padmai beel of Netrakona. Therefore, the concentrations of ammonia-nitrogen in all experimental sites of the present study were within acceptable limits, and there were no significant variations among the treatments. The values of chlorophyll $a\left(\mu \mathrm{g} \mathrm{l}^{-1}\right)$ indirectly expressed the abundance of phytoplankton of the water body. Ahmed (1993) found a negative relationship between chlorophyll $a$ and water transparency. Khatrai (1984) also found a positive relationship between phytoplankton and chlorophyll $a$. In the present study, chlorophyll $a$ values ranged from 28.80 to $234.88 \mu \mathrm{g}$ $1^{-1}$ and the mean values in treatments 1,2 and 3 were
$63.3727 \pm 5.94331, \quad 87.3987 \pm 32.91639 \quad$ and $63.5733 \pm 6.98469 \mu \mathrm{g} \mathrm{l^{-1 }}$, respectively. These values indicate that all the experimental sites were highly productive and suitable for fish production. The ranges of total alkalinity in present study found from $117 \mathrm{mg} \mathrm{l}^{-1}$ to $148 \mathrm{mg} \mathrm{l}^{-1}$. These values are similar with the findings of Khan et al. (1990).

During the study period, a total of 43 genera of phytoplankton (Bacillariophyceae, Chlorophyceae, Cyanophyceae, Euglenophyceae) and 9 genera of zooplankton (Crustacea and Rotifera) were identified. Most dominant genera were Fragillaria, Navicula, Nitzchia, Melosira, Ankistrodesmus, Chlorella, Pediastrum, Scenedesmus, Spirogyra, Tetraedron, Ulothrix, Microcystis, Gomphosphaeria, Oscillatoria, Euglena,, Brachionus, Asplanchna, Notholca, Lecane, Keratella, Moina, Cyclops, and Nauplius. Singh et al. (1999) identified 31 genera of phytoplankton and 14 of zooplankton. Total zooplankton was significantly higher in treatment $\mathrm{T}_{3}$ might be due higher organic decomposition and lower current.

A total of 32 species were recorded from different treatments viz. 30 from Matshyarani fish sanctuary and 25 and 24 species from $1 \mathrm{~km}$ upstream and downstream of the sanctuary, respectively. Khosru (2007) reported 25 species inside of the sanctuary and 30 species from outside of the sanctuary. So the number of species increased after establishment of sanctuary. The highest numbers of species were found in the last two months - April and May and the least species were found in the first month of the sampling - January might be due to fishes were took time to adapt in the new habitat. The most abundant SIS in number were Macrobrachium rude (Kucha chingri), Puntius ticto (Tit punti), Mystus bleekeri (Gulsha), Mystus vittatus (Tengra) and Macrobrachium malcolmsonii (Chotka chingri) in $\mathrm{T}_{1}$; Macrobrachium rude (Kucha chingri), Puntius ticto (Tit punti), Glossogobius giuris (Baila), Puntius sophore (Jat punti) and Mystus bleekeri (Gulsha) in $\mathrm{T}_{2}$ and Macrobrachium rude (Kucha chingri), Puntius ticto (Tit punti), Salmostoma phulo (Chela), Glossogobius giuris (Baila) and Puntius sophore (Jat punti) in $\mathrm{T}_{3}$. Both number of species and individual fish gradually increased over the months in the Matshyarani sanctuary than the other sites of the present study, as more and more periphyton and other food materials grown on the tree branches and bamboos and the habitat became more suitable for the fish. Haque et al. (2007) observed 43 species with the highest availability of a loach - Psilorhynchus sucatio and a Bagrid catfish, Tengra - Mystus vittatus in the three sanctuaries established under CBFM-2 project in the three rivers namely - Updakhali, the Kalihar 
and the Kangsha in Netrokona district using bamboo and tree branches as sanctuary materials.

The reported endangered fishes were reappeared in the sanctuary - the notable were kanchan putiPuntius conchonicus, Balichata- Acanthocobitis botia, Amblyceps mangois, Chaka- Chaca chaca, chhep chela- Chela cachius, bheda- Nandus nandus, gang gutum- Lepidocephalichthys irrorata, etc species were found in different treatments throughout the study period whereas kanchan puti- Puntius conchonicus, Balichata- Acanthocobitis botia, Amblyceps mangois, Chaka- Chaca chaca species were only found in Matshyarani fish sanctuary. The availability of these species were highest in sanctuary, because of its potentiality, food availability, shelter for free breeding and hazardless environment. Kucha chingri was abundantly available in all the samplings and stood first regarding the number of individuals. The abundance of minnows and kucha chingri proved that the fish sanctuary was especially helpful and acted as friendly ecosystem for small fishes than the other sites.

\section{Conclusions}

Setting up of a fish sanctuary can help to improve the status of fish habitat and biodiversity in perennial rivers like the old Brahmaputra which became a seasonal rivulet due to many manmade and natural factors. The setting up of a sanctuary in the deeper part of the Brahmaputra where fish and other aquatic animals can stay during the lean season and grow and attain maturity for spawning in the next monsoon- is obviously very significant. At the onset of next monsoon, these aquatic animals are expected to be dispersed on different part of the river and contributing the recruitments from the next year. The SIS abundance is related to natural habitat as well as the water quality and food abundance in the river.

The present study revealed that the qualitative and quantitative SIS diversity and natural food abundance inside the Matshyarani fish sanctuary were significantly more than both the upstream and downstream of the sanctuary. Sanctuary is an important fisheries management tool is used worldwide for the conservation, protection and restoration of fish species.

\section{References}

Ahmed, Z. F. 1993. Electivity index and dietary overlap of Catla catla (Hamilton) in fertilized ponds of Bangladesh. M. S. Thesis, Department of Fisheries Biology and Limnology, Bangladesh Agricultural University, Mymensingh. 163p.
APHA. 1992. Standard Methods for the Examination of the Water and Wastewater. American Public Health Association, Washington DC, USA. 874p.

CIFRI. 2000. River Godavari- Environment and Fishery. Bull. No. 102. The Director, CIFRI, Barrackpore-743 101, West Bengal. Calcutta Laser Graphics (Pvt.) Ltd. Calcutta 700006. 29p.

DoF (Department of Fisheries). 1996. Technologies and Management for Fisheries Development. Fisheries Fortnight-compendium, Ramna, Dhaka. Pp. 21-148.

Haque M., Ahmed, M. and Mustafa, M.G. 2007. Preferences of fishes to different types of Katha materials used in sanctuaries in three rivers of Netrokona, Bangladesh. Bangladesh J. Fish. (Special Issue, 2007) 30:147-156.

Hoq, M. E., Wahab, M. A. and Islam, M. N. 2006. Hydrographic status of Sundarbans mangrove, Bangladesh with special reference to post-larvae and juveniles fish and shrimp abundance. Wet Ecol. and Man. 14: 79-93.

Hossain, M. A. and Afroze, S. 1991. Small Fish as a resource in rural Bangladesh. Fishbyte. 9(2): 1618.

Hossain, M. A., Afsana, K. and Azad Shah, A.K.M. 1999. Nutritional value of some Small Indigenous fish Species (SIS) of Bangladesh. Bangladesh J. Fish. 3(1): 16-20.

Khan, M.A., Srivastava, K.P. , Dwivedi, R.K., Singh, D.N., Tyagi R.K. and Mehrotra, S.N. 1990. Significance of ecological parameters in fisheries management of a newly impounded reservion. Bachhara veservoir In: A.G. Jhingran, U.K. Unnithan and A. Gosh (eds).

Khatrai, T. C. 1984. Seasonal variation in the ecosystem of Lakhotia lake in Rajastan. India $J$. Fish. 3 (1): 122-129.

Khosru, M. S. 2007. Impact of Matshyarani sanctuary on fish biodiversity and socio-economic status of fishers. A thesis submitted to the department of Fisheries Biology and Genetics, Bangladesh Agricultural University, Mymensingh. 78p.

Nath, D. and Srivastava, N.P. 2001. Physico-chemical characteristics of Narmada for the stretch Sandia to Mola in M.P. state in the context of construction of reservoirs on the River or its tributaries. J. Inland Fish. Soc. India, 33(1): 1724.

Pathak, V., Mahavar, L. R. and Sarkar, A. 2001. Ecological status and production dynamics of a stretch of River Mahanadi. J. Inland Fish. Soc. India. 33(1): 25-31.

Rahman, M. M. 2000. Comparison of benthic fauna of two beels of Netrakona district under management condition. MS Thesis, Department 
of Aquaculture. Bangladesh Agricultural University, Mymensingh. 93p.

Shafi, M. and M. M. A. Quddus. 1982. Bionomics of the common punti, Puntius stigma (Cuv. and Val.) of Bangladesh. Bangladesh J. Zool., 2(1): 77-82.

Singh, D. N., Das, A. K., Shrivastava, N. P. and Sukumaran, P. K. 1999. Status of plankton in River Godavari. In: M. Sinha, D. kumar and P.K. Katiha(eds.). Eco-Friendly Management of Resources for Doubling Fish Production-
Strategies for $21^{\text {st }}$ Century. Proceedings of the National Seminar. IFSI, Barrackpore, India. pp. 128-132.

Tucker, C. S. and Boyd, C. E. 1979. Water quality. In: C. S. Tucker (ed.), Channel Catfish Culture. Elsevier Sci. Publ. Co., Amsterdam, the Netherlands. pp. 35-22.

Whitefield A. K., Blaber, S. J. M. and Cyrus D. P. 1981. Salinity ranges of some South African fish species occurring in estuaries. S. Afr. J. Zool. 16: 151-155. 